\title{
Cerebral Asymmetry of the "Top-Down" Allocation of Attention to Global and Local Features
}

\author{
Shuhei Yamaguchi, Shingo Yamagata, and Shotai Kobayashi \\ Department of Internal Medicine III (Neurology), Shimane Medical University, Izumo 693-8501, Japan
}

Neuropsychological and neuroimaging studies have indicated that global and local features of a visual scene are processed differentially by the two hemispheres. Although visual selective attention is suggested to play a role in the hemispheric asymmetry, the precise mechanism of attentional control for the global-local processing is not fully understood. We investigated the neural correlates of cerebral asymmetry in directing attention to the global pattern and local components of a hierarchical stimulus. Event-related brain potentials (ERPs) were recorded while the subjects shifted their attention to the global or local level of a hierarchically structured letter. The shift direction was controlled by a preceding cue stimulus. The ERPs to the cue stimuli demonstrated that the right temporal-parietal and left posterior temporal regions were differentially activated during attentional allocation to the global and local levels, respectively. These effects started $240 \mathrm{msec}$ after the cue stimulus. The late ERP components to the target stimuli also manifested the hemispheric global-local asymmetry as seen during the attentional shift. These electrophysiological results provided an asymmetrical neural basis for the "top-down" allocation of attention to global and local features and revealed the contribution of the temporal-parietal cortex to this attentional mechanism.

Key words: cerebral asymmetry; global and local attention; attention shift; hierarchical stimulus; event-related brain potential; temporal-parietal cortex; current source density
Hemispheric asymmetry associated with human perception of hierarchically organized visual scenes has received considerable attention (Sergent, 1982; Robertson and Lamb, 1991; Brown and Kosslyn, 1993; Ivry and Robertson, 1998). Regarding perceptual representation, the most striking difference is at the level of the scale for encoding objects. The left hemisphere is relatively better at encoding component parts (local level), whereas the right hemisphere is relatively better at encoding overall patterns (global level). Lesion studies have demonstrated that damage to the posterior superior temporal gyrus in the right hemisphere results in selective difficulty in identifying features at the global level, whereas homologous lesions in the left hemisphere result in selective difficulty in identifying objects at the local level (Robertson et al., 1988; Lamb et al., 1990).

Psychological experiments have suggested that the allocation of attention to particular spatial frequencies might play an important role in the hemispheric asymmetry (Kitterle et al., 1990; Christman et al., 1991). When a subject is required simply to detect a target within a compound stimulus, no difference is noted in the response time to high- or low-frequency stimuli presented in the right or left visual fields. On the other hand, when the subjects are asked to discriminate the target stimuli, the reaction times are faster for high-frequency stimuli presented in the right visual field and for low-frequency stimuli presented in the left visual field.

A positron emission tomography (PET) study demonstrated distinct foci of brain activation during sustained attention to global and local features (Fink et al., 1996). Directing attention

Received Jan. 14, 2000; revised Feb. 25, 2000; accepted Feb. 25, 2000.

This work was supported by Japanese Ministry of Education, Science, Sports and Culture Grants 11670626 to S.Y. and 10670586 to S.K. and a grant from the Japanese Ministry of Health and Welfare to S.K. We thank R. T. Knight for comments on this manuscript.

Correspondence should be addressed to Dr. Shuhei Yamaguchi at the above address. E-mail: yamagu3n@shimane-med.ac.jp.

Copyright (C) 2000 Society for Neuroscience $0270-6474 / 00 / 200001-05 \$ 15.00 / 0$ toward the global form resulted in activation of the right lingual gyrus, whereas locally directed attention activated the left inferior occipital cortex. Furthermore, this study showed that the temporal-parietal area plays a supervisory role in attentional control for global and local processing within the prestriate cortex. This "top-down" attention mechanism appears consistent with the neuropsychological data (Robertson et al., 1988). However, PET technology did not provide precise information about the relationship between attention-switching processes and hemispheric asymmetry. The time course of cortical activation related to attentional allocation was also difficult to delineate using PET or functional magnetic resonance imaging (MRI) (Fink et al., 1997; Martinez et al., 1997).

We addressed these issues by recording event-related brain potentials (ERPs) with high-density electrode arrays during a task requiring an attention shift, triggered by a cue stimulus preceding a hierarchical letter stimulus. A cueing study is suitable for examining neural processes related to the attention shift itself (Robertson et al., 1993; Kotchoubey et al., 1997), although most previous ERP studies have dealt with the processing of global and local features per se (Heinze and Munte, 1993; Johannes et al., 1996; Heinze et al., 1998; Proverbio et al., 1998; Han et al., 1999).

This article is published in The Journal of Neuroscience, Rapid Communications Section, which publishes brief, peerreviewed papers online, not in print. Rapid Communications are posted online approximately one month earlier than they would appear if printed. They are listed in the Table of Contents of the next open issue of JNeurosci. Cite this article as: JNeurosci, 2000, 20:RC72 (1-5). The publication date is the date of posting online at www.jneurosci.org.

http://www.jneurosci.org/cgi/content/full/4136 


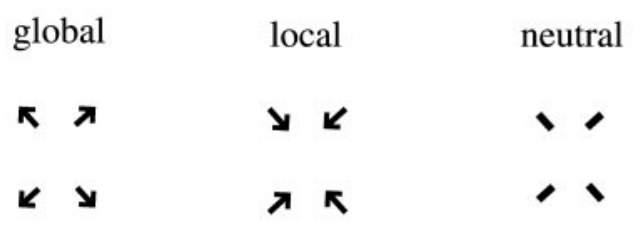

$\begin{array}{lrlrll}\mathrm{H} & \mathrm{H} & \mathrm{S} & \mathrm{S} & \mathrm{H} H H H H & \text { SSSSS } \\ \mathrm{H} & \mathrm{H} & \mathrm{S} & \mathrm{S} & \mathrm{H} & \mathrm{S} \\ \mathrm{H} H H H \mathrm{H} & \text { SSSSS } & \text { HHHHH } & \text { SSSSS } \\ \mathrm{H} & \mathrm{H} & \mathrm{S} & \mathrm{S} & \mathrm{H} & \mathrm{S} \\ \mathrm{H} & \mathrm{H} & \mathrm{S} & \mathrm{S} & \text { HHHHH } & \text { SSSSS }\end{array}$

Figure 1. Drawings of the cue and compound stimuli used in the present experiment.

The current study provided electrophysiological evidence for lateralized hemispheric specialization for allocation of visual attention to global and local features; the left temporal-parietal and right temporal cortical regions were activated differently by shifting attention to global and local levels, respectively.

\section{MATERIALS AND METHODS}

Subjects. The subjects were 16 normal, right-handed (Oldfield, 1971) volunteers (15 males and 1 female) recruited from our university population. The volunteers ranged from 25 to 40 years of age, and all had normal or corrected-to-normal visual acuity. Informed consent was obtained before the experiment.

Stimuli. The subjects were seated in a chair in an electrically shielded, sound-attenuated room with dimmed lights. The stimuli, which were white images presented on a black background, were presented on a 20 -inch color monitor placed $70 \mathrm{~cm}$ from the subject's eyes. The experiment consisted of 620 trials. Each trial began with the presentation of a cue, followed by a compound stimulus (Fig. 1). The cue consisted of four bars with or without an arrowhead placed at each corner of an imaginary rectangular frame, subtending $1.3^{\circ}$ (horizontal) $\times 1.7^{\circ}$ (vertical) of visual angle. This visual angle was intermediate between the global and local letters forming a compound stimulus. There were three types of cues: outward-pointing arrowheads (global cue), inward- pointing arrowheads (local cue), and oblique bars without arrowheads (neutral cue). The cue remained on the monitor for $800 \mathrm{msec}$ until the presentation of a compound stimulus. A compound stimulus consisted of a global letter $(\mathrm{H}$ or $\mathrm{S}$ ) made up of local letters $(\mathrm{H}$ or $\mathrm{S})$ in a $5 \times 5$ matrix. The global and local letters subtended $2.5 \times 3.5$ and $0.35 \times 0.5^{\circ}$ of visual angle, respectively. The compound stimulus remained on the monitor for 100 msec. The interstimulus interval between the compound stimulus and next cue was $1100 \mathrm{msec}$, during which time no stimulus was present on the monitor. There was no central fixation mark on the monitor, because a central fixation mark may require subjects to adjust their attention to spatial frequencies other than those of the cue and compound stimuli.

Procedure. A go/no-go task was adopted in this study. The subjects were instructed to press a button with the right index finger as quickly and accurately as possible when a target letter ( $\mathrm{H}$ or $\mathrm{S}$ ) was presented at the hierarchical level designated by cue stimuli. When the cue stimulus was neutral, the subjects had to respond to target stimuli presented at either hierarchical level. The assignment of the target letter (H or S) was counterbalanced across subjects before the experiment. The global cue indicated that the subjects should attend only to the global letter and ignore the local letter. The local cue indicated that the local letter should be identified and the global letter ignored. The neutral cue provided no information regarding the target level to be attended. The cues were delivered in a pseudorandom order with probabilities of $29 \%$ for the global and local cues, respectively, and $42 \%$ for the neutral cue. After the global cue was presented, the target letter appeared at both levels (congruent trial) or only at the global level (global incongruent trial) with an equal probability ( $12.5 \%$ among all of the trials). In some trials, a nontarget letter appeared at both levels with a lower probability $(4.0 \%$, no-go trial). For the local cue trial, the same probabilities were applied, except the target letter was at the local level for the incongruent trial (local incongruent trial). The neutral cue was followed by one of four types of compound stimuli, congruent $(12.5 \%)$, global incongruent $(12.5 \%)$, local incongruent $(12.5 \%)$, or nontarget trials $(4.5 \%$, no-go trial). To obtain enough electrophysiological data for target stimuli, the probability of nontarget (no-go) trials was relatively low as a consequence (12.5\% among all of the trials).

ERP recording and data analysis. ERPs were recorded from the scalp using a 128-channel electroencephalograph (EEG) net (Electrical Geodesics, Inc., Eugene, OR). The scalp locations of these channels are shown in Figure 2, bottom left corner. All of the electrodes were referenced to the vertex channel during the recording. The EEG samples were amplified with a $0.01-50 \mathrm{~Hz}$ bandpass filter, digitized at a rate of 250 samples/sec, and stored on a hard disk for off-line analysis. The EEGs were averaged over $1200 \mathrm{msec}$, time-locked to the cue and target, for each condition separately, including $200 \mathrm{msec}$ of a prestimulus baseline. Individual trials with excessive muscle activity ( $>70 \mu \mathrm{V}$ peak-to-peak) or eye movement $(>70 \mu \mathrm{V}$ peak-to-peak amplitude) were excluded. The averaged data were rereferenced algebraically to an averaged value across all electrodes.

We separated the electrode array into eight groups (lateral frontal, medial frontal, anterior temporal, central, posterior temporal, parietal, lateral occipital, and medial occipital), and ERPs within each electrode group were collapsed for the statistical analyses (see Fig. 2). The mean amplitudes were calculated at $20 \mathrm{msec}$ intervals between the cue and target onset with reference to the $200 \mathrm{msec}$ prestimulus baseline. To obtain neural activity related to the shift of attention to global and local features, we subtracted the ERPs to the neutral cue from those to the global and local cues. This procedure could eliminate ERPs related to neural activities such as primary sensory responses to the cue stimulus or general responses in preparation for the target stimulus. For each scalp site, the results were evaluated statistically using an ANOVA for repeated measures using cue type (global and local) and hemisphere (right and left) as independent factors. To locate neural sources responsible for the differential hemispheric activities associated with shifting attention to global and local features, we analyzed the intensity of the current source activities using low-resolution electromagnetic tomography (LORETA) (Pascual-Marqui et al., 1994). This analysis yielded all possible threedimensional distributions of current density in a three-shell head model without any preexisting assumptions concerning the number of sources.

We also analyzed ERPs to the target stimuli. Four major components were identified. Their peak amplitudes were measured relative to the prestimulus baseline, and peak latencies were measured relative to the target onset. The measurement windows were $100-160 \mathrm{msec}$ for P1 (postitive) and 180-250 msec for N1 (negative) at the medial occipital sites (Fig. 2, areas $h, l$ ) and 250-350 msec for N2 and 350-550 msec for $\mathrm{P} 3$ at the parietal sites (Fig. 2, areas $g, k$ ). The reaction time (RT) data were also analyzed using ANOVA for repeated measures. The Bonferroni-Dunn procedure was used for post hoc comparisons of the mean values.

\section{RESULTS}

\section{Psychophysics}

Behavioral data showed that an informative cue (global and local cue) provoked a shift of attention to the designated level. The RTs after the neutral cue were significantly slower than those after the global and local cues [neutral cue, $465 \pm 11$ (mean \pm SE) msec; global cue, $418 \pm 11 \mathrm{msec}(p<0.001$, vs neutral cue); local cue, $435 \pm 9 \mathrm{msec}(p<0.05$ vs neutral cue $)]$. Consistent with previous reports (Navon, 1977; Van Kleeck, 1989; Robertson and Lamb, 1991), the data showed global precedence and congruency effects. The RTs to global targets following the global cue were significantly faster than those to local targets following the local cue $(p<0.05)$. The congruency between global and local levels of hierarchical stimuli facilitated the response speed for all conditions [difference between congruent and incongruent trials, $27 \pm 5 \mathrm{msec}$ for global cue $(p<0.001)$; $19 \pm 5 \mathrm{msec}$ for local cue $(p<0.005) ; 72 \pm 5 \mathrm{msec}$ for neutral cue $(p<0.0001)]$. Thus, there was a significant interaction between cue type and congruency ( $p<0.0001)$, indicating that congruency effects were much larger for the neutral cue, compared with the global and 

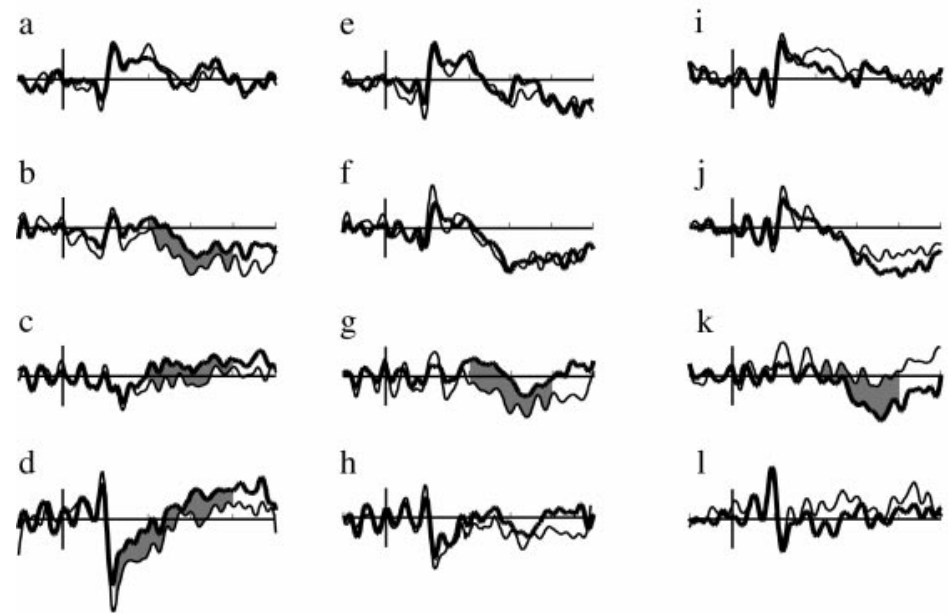

h
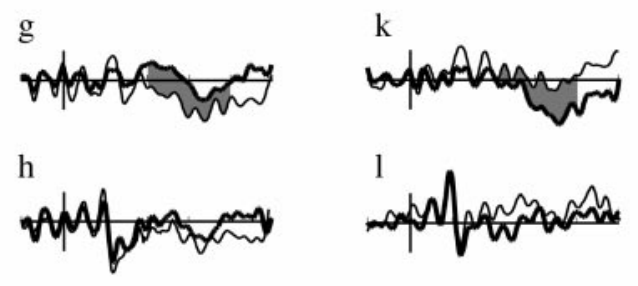

global cue - neutral cue

local cue - neutral cue
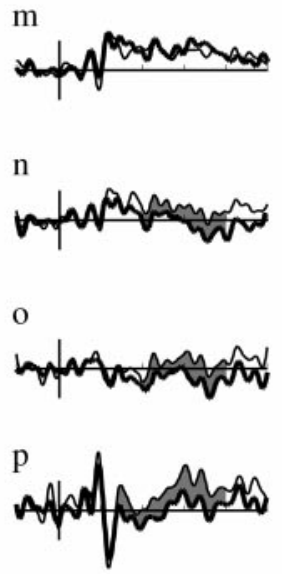

n
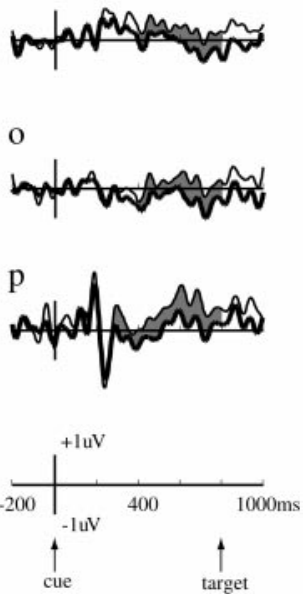

Figure 2. Electrophysiological indices of the attention shift to global and local features. Two subtracted grand averaged ERPs to cue stimuli were compared; the thick line is for a global shift (global cue - neutral cue), and the thin line is for a local shift (local cue - neutral cue). The shaded area indicates that significant interactions were observed between shift direction and hemisphere. Bottom left corner, Arrangement of the high-density electrode arrays and the locations of the grouped electrodes $(a-p)$ over the skull. a) Global shift
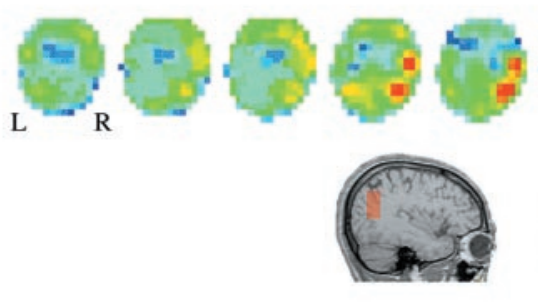

b) Local shift

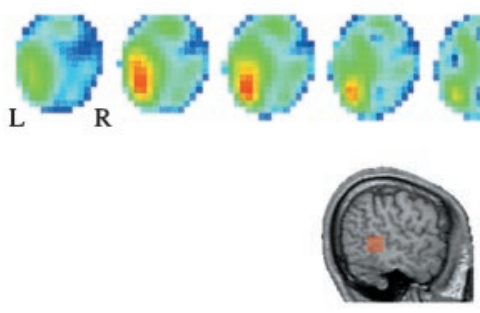

0

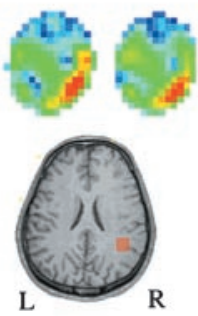

$\mathrm{L}$

$\mathrm{R}$
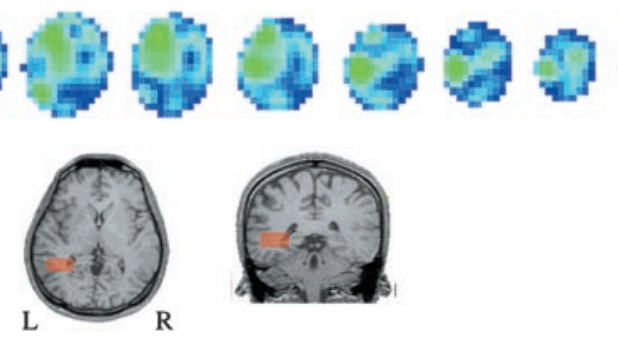

Figure 3. LORETA maps of the current source density of neural activity for attention shifts to global $(a)$ and local $(b)$ levels. The averaged data from 16 subjects were used for the analysis in the time window between 500 and $700 \mathrm{msec}$ after the cue. The maps are shown for successive horizontal slices from $2 \mathrm{~cm}$ (left) to $14 \mathrm{~cm}$ (right) superior to a plane containing the nasion and bilateral preauricular points. The areas showing a maximal current source are superimposed on a representative MRI for each shift condition. local cues. For the incongruent trials there was no interaction between target level (global vs local) and cue type (global or local cue vs neutral cue), indicating that global precedence was observed for both the neutral and informative cues to the same degree.

\section{ERPs to cue stimuli}

To examine differential hemispheric activation during attention shifts, we assessed the interaction between cue level (global vs local) and hemisphere (right vs left) on the ERP difference waveforms (global - neutral vs local - neutral) (Fig. 2). This revealed that the ERP is more negative over the right hemisphere during the shift of attention to the global level, whereas the ERP is more negative over the left hemisphere during the shift of attention to a local level. We determined the onset of the interaction by examining consecutive $20 \mathrm{msec}$ intervals. No interactions were observed between attention level and hemisphere until
$240 \mathrm{msec}$ after the cue onset. Subsequently, the interaction commenced in the lateral occipital regions (areas d and p; Fig. 3, shaded). This interaction continued until the target appeared (the interaction was analyzed during the cue-target interval). Following the lateral occipital region, similar interactions also began in the parietal (areas $\mathrm{g}$ and $\mathrm{k}$ ), posterior temporal (areas c and o), and anterior temporal (areas b and $\mathrm{n}$ ) regions after $400 \mathrm{msec}$. All of the interactions continued until the targets appeared. Other anterior parts of the scalp showed no interactions between cue level and hemisphere during the cue-target interval.

To confirm the differential hemispheric activation caused by shifting attention to global and local features, we obtained topographical maps of the source activities for processing global and local cues using the LORETA method. The two subtracted ERPs were analyzed in the time window between 500 and $700 \mathrm{msec}$ after the cue, during which interval the hemispheric difference 
a

P1 at the medial occipital site

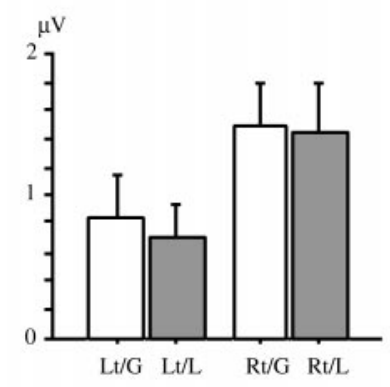

c

N2 at the parietal site *

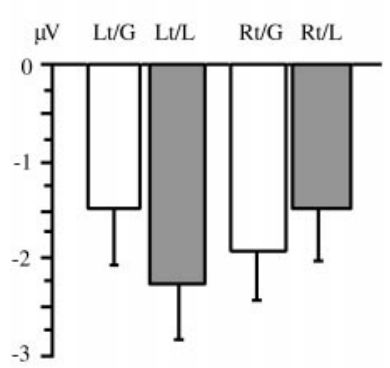

b

N1 at the medial occipital site

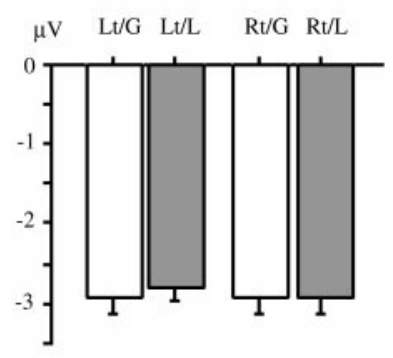

d P3 at the parietal site **

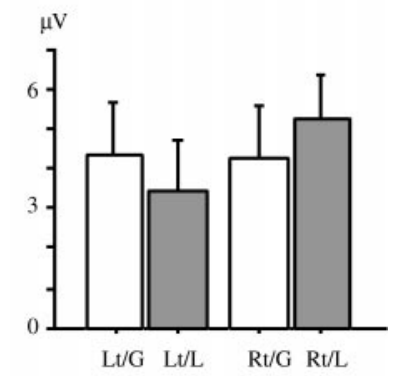

Figure 4. Mean amplitude of each ERP component to target stimuli as functions of the hemisphere and cue level. The data were analyzed at the medial occipital site for $\mathrm{P} 1(a)$ and $\mathrm{N} 1(b)$, at the lateral occipital site for $\mathrm{P} 2(c)$, and at the parietal site for N2 $(d)$ and P3 (e). Lt/G, Recorded in the left hemisphere for global targets; $R t / G$, recorded in the right hemisphere for global targets; $L t / l$, recorded in the left hemisphere for local targets; Rt/l, recorded in the right hemisphere for local targets. $*, * *$ Significant interaction between hemisphere and cue level (* $p<0.005$; $* * p<0.02)$.

was most prominent (see Fig. 2). A dense current source was observed in the right temporal-parietal area with an attention shift to the global level, whereas a strong current source was seen in the left posterior temporal region with an attention shift to the local level (Fig. 3). Thus, the asymmetric surface potentials resulted from neural activity in the right temporal-parietal area for a global attention shift and activity in the left posterior temporal area for a local attention shift.

\section{ERPs to target stimuli}

Finally, we analyzed ERPs to target stimuli to clarify whether a hemispheric difference was also manifested during processing of global and local stimuli per se. For this analysis, we compared the ERPs to congruent global and local targets after the informative cues. The P1 component was larger over the right occipital site than over the left side $(p<0.05)$ without an interaction between target level and hemisphere (Fig. 4a). There was neither hemispheric asymmetry nor an interaction for the posterior N1 (Fig. $4 b$ ). On the other hand, the N2 component demonstrated an interaction between target level and hemisphere $(p<0.005$; Fig. $4 c$ ). This effect was attributable to enhanced amplitude over the left hemisphere for local targets compared with global targets, whereas the amplitude was larger for global targets compared with local targets over the right hemisphere. The P3 component showed the same interaction as observed for the N2 $(p<0.02$;

Fig. 4d); the P3 was negatively shifted for local targets relative to global targets over the left hemisphere, whereas the amplitude was more negative for global targets compared with local targets over the right hemisphere. Thus, a similar hemispheric difference was observed in the relatively late ERP components related to processing of global and local features per se, as seen in the components related to the shifting of attention. The analysis of the latencies revealed that only the P3 latency to global targets was significantly shorter than that to local targets $(411 \pm 37$ vs $436 \pm 36 \mathrm{msec} ; p<0.05)$ whereas other components showed comparable latencies between the global and local targets.

\section{DISCUSSION}

Ample evidence for hemispheric asymmetry in global-local feature processing has been provided by various neuroimaging techniques (Fink et al., 1996; Martinez et al., 1997; Heinze et al., 1998; Proverbio et al., 1998; Han et al., 1999). The current study demonstrated that differences in neural activities between global and local processing first appeared $250 \mathrm{msec}$ after stimuli (i.e., N2). The N2 ERP component showed a relatively larger amplitude in the left hemisphere for local targets and in the right hemisphere for global targets. This is consistent with previous ERP studies (Heinze and Munte, 1993; Heinze et al., 1998), which demonstrated that the posterior negativity peaking at 250 msec for global and local targets had a similar onset latency but a different topography, suggesting that separate brain systems process global and local information in parallel. On the other hand, the sensory components (i.e., P1) of visual evoked potentials produced by various spatial frequencies showed consistently larger amplitudes over the right occipital site than over the left side, irrespective of the stimulus spatial frequency (Grabowska and Nowicka, 1996). This is also in line with our data and strongly suggests that the hemispheric lateralization of early visual perception does not depend on the stimulus spatial frequency.

The main focus of our study is how the attentional allocation to global or local features affects hemispheric specialization of global-local processing. The principal advantage of ERP over methods such as PET is its greater temporal resolution for delineating time-dependent mental processes. Our data demonstrated that the ERP segregation between attention shifts to global form and local elements emerged at $\sim 240 \mathrm{msec}$ after delivering the cue stimulus. This interval includes the time required to discriminate the level to be attended according to cue type. Interestingly, this time window is close to the time for cue-induced voluntary shifting of spatial attention (Yamaguchi et al., 1994).

The important aspect demonstrated in our study is that the hemispheric asymmetry arose not only during the task in which global-local processing was actually performed but also during the time interval in which attention was allocated to global or local levels. In addition to the hemispheric asymmetry during "bottom-up" processing, the current study directly demonstrated neural substrates for a top-down mechanism of hemispheric asymmetry in global and local selection. These neural activities were located in the temporal-parietal cortices in the right hemisphere for the global shift and in the posterior temporal cortices in the left hemisphere for the local shift. This hemispheric asymmetry is consistent with the neuropsychological evidence (Robertson et al., 1988; Lamb et al., 1989). Lesions centered in the left inferior parietal lobe did not disrupt local processing, whereas lesions centered in the left superior temporal gyrus did. Lesions including both the right superior temporal gyrus and inferior parietal lobe disrupted global processing. Previous PET results 
also showed the activation of these association cortices during a task associated with switching attention between global and local levels, although the neural activation in each hemisphere observed in the PET study could not be linked to global and local processing separately because of the paradigm used and the low temporal resolution (Fink et al., 1996). According to the present study, the laterality of neural activity associated with an attention shift corresponded to the asymmetry observed in brain activity during global and local processing per se. Our data support the hypothesis that hemispheric asymmetry is derived from descending influences of the right temporal-parietal and left posterior temporal regions, and these cortices may play a critical role in mediating the voluntary distribution and maintenance of selective attention. In addition, the current ERP data during target detection suggest that this attention mechanism does not direct the early sensory phase of processing. Instead, higher cognitive stages associated with late ERP components (i.e., N2 and P3) may be linked with such an allocation of attention.

Global precedence is another issue to be addressed in globallocal processing. The behavioral data demonstrated a global precedence in responding to a hierarchical stimulus in a selective attention task on a trial-by-trial basis. Electrophysiological correlates of global precedence have been demonstrated in a sustained focused attention task (Proverbio et al., 1998), indicating that faster and larger responses of the N180 (170-210 msec) and P3 (340-520 msec) components were associated with the global precedence effect. Another study also demonstrated that the RT advantage was reflected in the P3 latency (Heinze and Munte, 1993). Partially in agreement with those reports, we also found an earlier P3 component to global targets compared with local targets. Taken together, it is plausible that global precedence may emerge at the postperceptual, later processing stage. One intriguing issue is whether the global precedence is influenced by the presetting of attention. The behavioral data indicated that the global precedence after informative cues was not significantly different from that after the neutral cue, indicating that global precedence does not seem to be affected by cue-related attentional modulation. Thus, it is unlikely that the global advantage in global-local processing is determined by attentional set; instead, it may be related to postperceptual processing independent of attentional preset.

In conclusion, our study provides the first electrophysiological evidence of hemispheric specialization during the allocation of attention to global and local features. The right temporal-parietal and left posterior temporal regions play separate roles in this top-down attention mechanism. This attentional modulation may affect neural activity during the later processing stages of global or local aspects of complex visual scenes.

\section{REFERENCES}

Brown HD, Kosslyn SM (1993) Cerebral lateralization. Curr Opin Neurobiol 3:183-186.

Christman S, Kitterle F, Hellige J (1991) Hemispheric asymmetry in the processing of absolute versus relative spatial frequency. Brain Cogn 16:62-73.

Fink GR, Halligan PH, Marchall JC, Frith CD, Frackowiack RSJ, Dolan
RJ (1996) Where in the brain does visual attention select the forest and the trees. Nature 382:626-628.

Fink GR, Halligan PW, Marshall JC, Frith CD, Frackowiak SJ, Dolan RJ (1997) Neural mechanisms involved in the processing of global and local aspects of hierarchically organized visual stimuli. Brain 120:1779-1791.

Grabowska A, Nowicka A (1996) Visual-spatial-frequency model of cerebral asymmetry: a critical survey of behavioral and electrophysiological studies. Psychol Bull 120:434-449.

Han S, Fan S, Zhuo Y (1999) Modulation of brain activities by hierarchical processing: a high-density ERP study. Brain Topogr 11:171-183.

Heinze HJ, Munte TF (1993) Electrophysiological correlates of hierarchical stimulus processing: dissociation between onset and later stages of global and local target processing. Neuropsychologia 31:841-852.

Heinze HJ, Hinrichs H, Scholz M, Burchert W, Mangun GR (1998) Neural mechanisms of global and local processing: a combined PET and ERP study. J Cogn Neurosci 10:485-498.

Ivry RB, Robertson LC (1998) The two sides of perception. Cambridge, MA: MIT.

Johannes S, Wieringa BM, Matzke M, Munte TF (1996) Hierarchical visual stimuli: electrophysiological evidence for separate left hemispheric global and local processing mechanisms in humans. Neurosci Lett 210:111-114.

Kitterle F, Christman S, Hellige J (1990) Hemispheric differences are found in the identification, but not the detection, of low versus high spatial frequencies. Percept Psychophys 48:297-306.

Kotchoubey B, Wascher E, Verleger R (1997) Shifting attention between global features and details: an event-related potential study. Biol Psychol 46:25-50.

Lamb MR, Robertson LC, Knight RT (1989) Attention and intereference in the processing of global and local information: effects of unilateral temporal-parietal junction lesions. Neuropsychologia 27:471-483.

Lamb MR, Robertson LC, Knight RT (1990) Component mechanisms underlying the processing of hierarchically organized pattern: inferences from patients with unilateral cortical lesions. J Exp Psychol Learn Mem Cogn 16:471-483.

Martinez A, Moses P, Frank L, Buxton R, Wong E, Stiles J (1997) Hemispheric asymmetries in global and local processing: evidence from fMRI. NeuroReport 8:1685-1689.

Navon D (1977) Forest before trees: the precedence of global features in visual perception. Cognit Psychol 9:353-383.

Oldfield RC (1971) The assessment and analysis of handedness: the Edinburgh inventory. Neuropsychologia 9:97-113.

Pascual-Marqui RD, Michel CM, Lehmann D (1994) Low resolution electromagnetic tomography: a new method for localizing electrical activity in the brain. Int $\mathbf{J}$ Psychophysiol 18:49-65.

Proverbio AM, Minniti A, Zani A (1998) Electrophysiological evidence of a perceptual precedence of global vs. local visual information. Cogn Brain Res 6:321-334.

Robertson LC, Lamb MR (1991) Neuropsychological contributions to theories of part/whole organization. Cognit Psychol 23:299-330.

Robertson LC, Lamb MR, Knight RT (1988) Effects of lesions of temporal-parietal junction on perceptual and attentional processing in humans. J Neurosci 8:3757-3769.

Robertson LC, Egly R, Lamb MR, Kerth L (1993) Spatial attention and cuing to global and local levels of hierarchical structure. J Exp Psychol Hum Percept Perform 19:471-87.

Sergent J (1982) The cerebral balance of power: confrontation or cooperation. J Exp Psychol Hum Percept Perform 8:253-271.

Van Kleeck MH (1989) Hemispheric differences in global versus local processing of hierarchical visual stimuli by normal subjects: new data and a meta-analysis of previous studies. Neuropsychologia 27:1165-1178.

Yamaguchi S, Tsuchiya H, Kobayashi S (1994) Electroencephlographic activity associated with shifts of visuospatial attention. Brain 117:553562. 